\title{
Estrutura e composição florística de cinco áreas de caíva no Planalto Norte de Santa Catarina
}

\author{
Ana Lúcia Hanisch¹, Gilcimar Adriano Vogt ${ }^{1}$, Anésio da Cunha Marques², Luís Cláudio Bona ${ }^{3}$, Daniel Darlon Bosse \\ ${ }^{1}$ Epagri, Estação Experimental de Canoinhas, BR 280, Km 219, CEP 89460-000, Canoinhas, SC, Brasil, analucia@epagri.sc.gov.br; \\ gilcimar@epagri.sc.gov.br; ${ }^{2} \mathrm{ICM}-\mathrm{Bio}$, Floresta Nacional de Três Barras, Comunidade da Barra Grande, CEP 89490-000, Três Barras, SC, \\ Brasil, anesio_marques@gmail.com; 33S-PTA, Contestado, Rua 13 de maio, 55, CEP 89400-00, Porto União, SC, Brasil, bona@aspta. \\ org.br; ${ }^{4}$ Universidade do Contestado - UnC, Roberto Elhke, 85, CEP 89460-000, Canoinhas, SC, Brasil, bosse_d@yahoo.com.br
}

\begin{abstract}
Resumo - Caívas são remanescentes de Floresta Ombrófila Mista, com diferentes densidades arbóreas, cujo estrato herbáceo é formado por pastagens nativas ou naturalizadas utilizadas para pastejo animal. Este trabalho avaliou a composição florística e a estrutura arbórea de cinco caívas bem estabelecidas. Foram utilizadas parcelas de um hectare em cada caíva, onde foram medidos e identificados todos os indivíduos com DAP $\geq 5 \mathrm{~cm}$ e calculados os parâmetros fitossociológicos de densidade, frequência, dominância e valor de importância. Verificou-se a ocorrência de 24 famílias e 52 espécies. As famílias com maior número de espécies foram Lauraceae, Myrtaceae, Aquifoliaceae e Sapindaceae; e as famílias com maior número de indivíduos foram Myrtaceae, Araucariaceae, Aquifoliaceae e Lauraceae. O índice de diversidade de Shannon indicou grande diversidade florística. O índice de Sorensen indicou alta similaridade entre as cinco caívas. As espécies que contribuíram para os maiores valores de importância foram: Araucaria angustifolia, Myrcia sp., Ocotea porosa, Cinnamodendron dinisii e Ilex paraguariensis. Esses resultados confirmam que as caívas representam uma importante reserva de espécies de árvore nativas, mesmo sendo manejadas há longo tempo.
\end{abstract}

Termos para indexação: Floresta Ombrófila Mista, uso e manejo sustentável, sistemas agroflorestais, conservação da biodiversidade.

\section{Structure and floristic composition of five caiva area in north plateau of Santa Catarina State, Brazil}

\begin{abstract}
Caivas are remnants areas of Mixed Ombrophylous Forest, whose inferior stratum is composed of native pastures used for animal consume. The aim of this work was to evaluate the structure and floristic composition of five caivas areas. Plots of one hectare were used. Every individual with $\mathrm{DBH} \geq 5 \mathrm{~cm}$ were measured and identified. The parameters of density, frequency, dominance, Importance Value (IV) were calculated. These results showed 24 families and 52 species. The families with the higher number of species were Lauraceae, Myrtaceae, Aquifoliaceae and Sapindaceae and with the higher number of individuals were Myrtaceae, Araucariaceae, Aquifoliaceae and Lauraceae. The Shannon diversity index value showed high floristic diversity in the caivas and the Sorensen index showed floristic similarity among the five caivas areas. Araucaria angustifolia, Myrcia sp. Ocotea porosa, Cinnamodendron dinisii and Ilex paraguariensis were the five most important species based on Importance Value. The results confirmed that the caivas areas represent an important reservoir of native tree species, although they were being management for a long time.
\end{abstract}

Index terms: Mixed Ombrophylous Forest, sustainable forest management, agroforestry systems, conservation of biodiversity.

\section{Introdução}

Caíva é a denominação regional no Planalto Norte de Santa Catarina para remanescentes de Floresta Ombrófila Mista (FOM) com diferentes densidades arbóreas, cujos estratos herbáceos são compostos por pastagem nativa ou naturalizada extensivamente pastejada (Hanisch et al., 2009). As caívas são originárias das antigas áreas de invernadas ou faxinais que se distribuíam pelo CentroSul do Paraná, Norte de Santa Catarina e Norte do Rio Grande do Sul (Chang, 1988).

A maior parte das caívas apresenta-se como fragmentos florestais de tamanhos variados nas propriedades rurais. O conjunto desses fragmentos na região contribui para a 
formação da paisagem característica das regiões norte de Santa Catarina e parte do centro-sul do Paraná, formando um mosaico de áreas de cultivo intercaladas por áreas de florestas. Apesar de não ser computada especificamente nos censos agropecuários, sua estimativa é possível através da avaliação das áreas citadas como "potreiros", aparecendo em 39\% dos estabelecimentos rurais e ocupando cerca de $70 \mathrm{mil}$ ha ou, aproximadamente, 13\% do território do Planalto Norte de Santa Catarina.

Com relação à caracterização das caívas, há uma escassez significativa de informações. Do ponto de vista produtivo, elas são muito peculiares, pois há uma forte inter-relação entre o estrato arbóreo, espécies forrageiras e animais. As árvores fornecem sombra para os animais e proteção para as forrageiras contra as geadas, permitindo que essas se mantenham verdes por mais tempo, facilitando a mantença dos animais (Hanisch et al., 2009). Em algumas situações, a pressão de pastejo pode se acentuar, levando os animais a consumir a brotação das árvores, prejudicando sua regeneração. No entanto, considerando-se que as caívas existem há muitas décadas com esse manejo, seu uso pode ser considerado uma estratégia de sucesso no uso e manejo sustentável dos remanescentes florestais, pois provavelmente, sem o objetivo da manutenção dos rebanhos, muitas dessas áreas já teriam desaparecido.

Do ponto de vista ambiental, sua existência contribuiu para a conservação de mananciais hídricos e podem ser utilizadas estrategicamente como opção de corredores ecológicos para a fauna local. Outro aspecto relevante é que muitas áreas de caíva fazem parte da reserva legal das propriedades. Neste sentido, conservar esses remanescentes florestais pode contribuir para a preservação do ecossistema de FOM, que atualmente é um grande desafio para todo o Sul do Brasil (Sanqueta et al., 2002).

Segundo Pimentel et al. (1992), em regiões onde a pressão antrópica é intensa, o estabelecimento e a manutenção de grandes áreas de reserva tem sido difícil, principalmente quando as áreas de vegetação natural já se encontram muito reduzidas e fragmentadas. Nessas situações, uma alternativa a ser considerada é a conservação de espécies nativas através de sistemas que aliem produção agrícola e conservação. Essa alternativa se ajusta em relação às caívas, pois o manejo sustentável do estrato herbáceo e sua utilização de forma racional pelos animais podem contribuir para a manutenção do estrato arbóreo.
A partir dessa alternativa, um conjunto de instituições regionais vem desenvolvendo trabalhos de pesquisa participativa em propriedades familiares da região, sobretudo quanto ao desenvolvimento de estratégias de manejo sustentável de áreas de caíva. Neste sentido, esta pesquisa teve por objetivo caracterizar a composição florística e a estrutura arbórea de cinco áreas de caíva a fim de buscar estratégias que contribuam para seu manejo racional.

\section{Material e métodos}

Este estudo foi desenvolvido no norte de Santa Catarina, em cinco áreas de caíva pertencentes aos municípios de Três Barras, Canoinhas, Bela Vista do Toldo e Major Vieira, indicadas pelas seguintes coordenadas geográficas: $26^{\circ} 23^{\prime} \mathrm{S}$ e $50^{\circ} 24^{\prime} \mathrm{W}$; $26^{\circ} 27^{\prime} \mathrm{S}$ e $50^{\circ} 17^{\prime} \mathrm{W}$; $26^{\circ} 23^{\prime} \mathrm{S}$ e $50^{\circ} 30^{\prime} \mathrm{W}$; $26^{\circ} 14^{\prime} \mathrm{S}$ e $50^{\circ} 21^{\prime} \mathrm{W}$; $26^{\circ} 12^{\prime} \mathrm{S}$ e $50^{\circ} 14^{\prime} \mathrm{W}$. Os solos das áreas avaliadas foram classificados como Latossolo Vermelho Distrófico típico (Sistema..., 1999). A altitude média das cinco propriedades é de $800 \mathrm{~m}$. A vegetação primitiva é de Floresta Ombrófila Mista.

As caívas avaliadas localizam-se em cinco propriedades rurais familiares, que possuem em comum o relevo plano, a utilização com rebanho bovino, o uso contínuo da caíva por mais de 30 anos e solos com características físicas e químicas semelhantes (elevados teores de alumínio trocável e matéria orgânica, acidez alta e baixa saturação por bases).

Em cada propriedade foi instalada uma parcela amostral de 1 ha $(50 \mathrm{~m} \times 200 \mathrm{~m})$, com dez subparcelas de $20 \mathrm{~m}$ x $50 \mathrm{~m}$. O tamanho das parcelas foi baseado em recomendações de Lamprecht (1964) e Sanqueta et al. (2002), que sugerem parcelas grandes para levantamentos estruturais em florestas naturais.

Todos os indivíduos arbóreos e arbustivos presentes nas parcelas, com diâmetro à altura do peito (DAP) igual ou superior a $5 \mathrm{~cm}$ foram medidos com uma suta dendrométrica e identificados. Não foram consideradas árvores mortas no presente levantamento. Para os indivíduos com troncos ramificados, foram medidos todos os troncos que apresentavam DAP igual ou superior a $5 \mathrm{~cm}$ e considerado cada tronco como um indivíduo.

A identificação botânica foi realizada, quando possível, em campo e em laboratório com a comparação do material coletado com exsicatas do Herbário da 
Universidade do Contestado, Campus Canoinhas, e com apoio de literatura (Reitz et al., 1978; Backes \& Irgang, 2004; Souza \& Lorenzi, 2005). A ordenação das famílias e gêneros foi baseada no Angiosperm... (2003) e a nomenclatura das espécies foi verificada nos arquivos do Missouri... (2010).

Para avaliar a diversidade nas áreas de caíva, foram utilizados os índices de Shannon (H') e o de equabilidade de Pielou (J') (Magurran, 1988). Para avaliar a similaridade florística entre as caívas foi utilizado o índice de Sorensen, que leva em consideração as diferenças no número de espécies de áreas que se quer comparar.
Para análise da estrutura horizontal da vegetação, foram determinados os parâmetros fitossociológicos densidade, dominância, frequência e valor de importância (Mueller-Dombois \& Ellenberg, 1974). Para os cálculos de determinação dos parâmetros fitossociológicos foi utilizado o Programa Fitopac 1.6 (Shepherd, 2006).

\section{Resultados e discussão}

No total das cinco áreas de caíva avaliadas, foi constatada a ocorrência de 24 famílias botânicas e 52 espécies (Tabela 1).

Tabela 1. Número de indivíduos por L2 de espécies arbóreas em cinco caívas (C).

\begin{tabular}{|c|c|c|c|c|c|c|c|}
\hline Família & Espécie & Nome popular & C 1 & $\mathrm{C2}$ & $\mathrm{C3}$ & $\mathrm{C4}$ & C5 \\
\hline \multirow[t]{2}{*}{ Anacardiaceae } & Lithraea brasiliensis Marchand & bugreiro & 15 & 1 & 0 & 22 & 0 \\
\hline & Schinus terebinthifolius Raddi & aroeira & 97 & 6 & 0 & 3 & 1 \\
\hline Annonaceae & Rollinia rugulosa Schltdl & ariticum & 0 & 3 & 16 & 2 & 0 \\
\hline \multirow[t]{4}{*}{ Aquifoliaceae } & Ilex brevicuspis Reissek & caúna & 49 & 2 & 13 & 4 & 4 \\
\hline & Ilex microdonta Reissek & congonha & 24 & 1 & 5 & 5 & 7 \\
\hline & Ilex paraguariensis A. St. Hil. & erva-mate & 130 & 9 & 44 & 4 & 29 \\
\hline & Ilex theezans Mart. ex Reissek & caúna-amarga & 26 & 0 & 0 & 0 & 1 \\
\hline Araucariaceae & Araucaria angustifolia (Bertol.) Kuntze & pinheiro & 13 & 22 & 166 & 207 & 19 \\
\hline Arecaceae & Syagrus romanzoffiana (Cham.) Glassman & jerivá & 0 & 0 & 0 & 2 & 0 \\
\hline \multirow[t]{2}{*}{ Asteraceae } & Gochnatia polymorpha (Less.) Cabrera & cambará & 4 & 0 & 0 & 0 & 0 \\
\hline & Vernonia discolor (Spreng.) Less. & vassourão-preto & 0 & 0 & 1 & 0 & 0 \\
\hline \multirow[t]{2}{*}{ Bignoniaceae } & Jacaranda puberula Cham. & caroba & 4 & 0 & 2 & 3 & 2 \\
\hline & Tabebuia avellanedae var. paulensis Toledo & ipê-roxo & 0 & 0 & 0 & 1 & 0 \\
\hline Canellaceae & Cinnamodendron dinisii (Schwacke) Occhioni & pimenteira & 76 & 20 & 58 & 32 & 23 \\
\hline Clethraceae & Clethra scabra Pers. & carne-de-vaca & 75 & 0 & 6 & 4 & 2 \\
\hline Combretaceae & Terminalia australis Cambess. & sarandi & 3 & 0 & 0 & 0 & 0 \\
\hline \multirow[t]{2}{*}{ Euphorbiaceae } & Sapium glandulosum (L.) Morong & leiteiro & 11 & 6 & 3 & 1 & 1 \\
\hline & Sebastiania commersoniana (Baill.) L.B. Sm. \& Downs & branquilho & 56 & 0 & 26 & 4 & 4 \\
\hline \multirow[t]{2}{*}{ Fabaceae } & Acacia plumosa Lowe & unha-de-gato & 0 & 0 & 1 & 0 & 0 \\
\hline & Lonchocarpus subglaucescens Mart. ex Benth. & timbó & 2 & 0 & 16 & 0 & 15 \\
\hline Lamiaceae & Vitex megapotamica (Spreng.) Moldenke & tarumã & 1 & 0 & 2 & 0 & 4 \\
\hline \multirow[t]{8}{*}{ Lauraceae } & $\begin{array}{l}\text { Cinnamomum sellowianum (Nees \& Martius ex Nees) } \\
\text { Kosterm. }\end{array}$ & canela-sebo & 1 & 0 & 0 & 0 & 0 \\
\hline & Cinnamomum vesiculosum (Nees) Kosterm. & pau-d'alho & 14 & 0 & 2 & 14 & 1 \\
\hline & Cryptocarya aschersoniana $\mathrm{Mez}$ & $\begin{array}{l}\text { canela-batalha ou } \\
\text { nhuntinga }\end{array}$ & 0 & 11 & 0 & 0 & 0 \\
\hline & Nectandra grandiflora Nees & canela-amarela & 1 & 0 & 0 & 0 & 0 \\
\hline & Nectandra megapotamica (Spreng.) Mez & canela-imbuia & 43 & 0 & 0 & 0 & 3 \\
\hline & Ocotea corymbosa (Meisn.) Mez & $\begin{array}{l}\text { canela-preta ou } \\
\text { fedida }\end{array}$ & 1 & 0 & 3 & 12 & 0 \\
\hline & Ocotea porosa (Nees) Barroso & imbuia & 64 & 3 & 18 & 4 & 58 \\
\hline & Ocotea puberula (Rich.) Ness & canela-guaicá & 2 & 1 & 3 & 1 & 1 \\
\hline
\end{tabular}


Tabela 1. Continuação.

\begin{tabular}{|c|c|c|c|c|c|c|c|}
\hline Família & Espécie & Nome popular & C 1 & $\mathrm{C2}$ & $\mathrm{C3}$ & $\mathrm{C} 4$ & $\mathrm{C5}$ \\
\hline & Ocotea sp. & canela & 8 & 0 & 0 & 2 & 4 \\
\hline & Persea venosa Nees \& Mart. ex Nees & pau-andrade & 0 & 0 & 0 & 0 & 1 \\
\hline Meliaceae & Cedrela fissilis Vell. & cedro-branco & 0 & 0 & 0 & 3 & 0 \\
\hline Myrsinaceae & Rapanea umbellata (Mart.) Mez. & capororoca & 1 & 0 & 0 & 6 & 0 \\
\hline \multirow[t]{8}{*}{ Myrtaceae } & Campomanesia xanthocarpa O. Berg & guabirova & 11 & 0 & 17 & 35 & 1 \\
\hline & Eugenia involucrata DC. & cerejeira & 1 & 0 & 0 & 0 & 0 \\
\hline & Eugenia uniflora L. & pitanga & 12 & 0 & 0 & 1 & 6 \\
\hline & Eugenia uvalha Cambess. & uvaia & 3 & 0 & 6 & 0 & 0 \\
\hline & Mosiera prismatica (D. Legrand) Landrum & cerninho & 2 & 0 & 0 & 78 & 0 \\
\hline & Myrcia laruotteana Cambess. & cambuim & 1 & 0 & 3 & 0 & 0 \\
\hline & Myrcia sp. & guamirim & 126 & 362 & 275 & 5 & 21 \\
\hline & Psidium coriaceum Mart. ex O. Berg & araçá & 0 & 0 & 0 & 2 & 0 \\
\hline Nyctaginaceae & Guapira opposita (Vell.) Reitz & maria-mole & 1 & 0 & 0 & 0 & 1 \\
\hline \multirow[t]{2}{*}{ Rosaceae } & Cydonia oblonga Mill. & marmeleiro & 20 & 5 & 0 & 2 & 0 \\
\hline & Prunus sellowii Koehne & pessegueiro-bravo & 3 & 0 & 0 & 1 & 0 \\
\hline \multirow[t]{2}{*}{ Rutaceae } & Zanthoxylum rhoifolium Lam. & juvevê & 14 & 0 & 0 & 9 & 0 \\
\hline & Zanthoxylum sp. & mamica-de-cadela & 7 & 1 & 7 & 5 & 0 \\
\hline Salicaceae & Casearia decandra Jacq. & guaçatunga & 10 & 0 & 47 & 30 & 1 \\
\hline \multirow[t]{3}{*}{ Sapindaceae } & $\begin{array}{l}\text { Allophylus edulis (A. St.-Hil., Cambess. \& A. Juss.) } \\
\text { Radlk. }\end{array}$ & vacum & 10 & 1 & 8 & 1 & 0 \\
\hline & Cupania vernalis Cambess. & cuvatã & 22 & 0 & 37 & 16 & 1 \\
\hline & Matayba elaeagnoides Radlk. & miguel-pintado & 0 & 0 & 0 & 0 & 1 \\
\hline Solanaceae & Acnistus breviflorus Sendtn. & espora-de-galo & 1 & 2 & 3 & 0 & 0 \\
\hline Winteraceae & Drimys brasiliensis Miers & cataia & 13 & 3 & 45 & 8 & 5 \\
\hline Total & & & 978 & 459 & 833 & 529 & 217 \\
\hline
\end{tabular}

As famílias com maior número de espécies na avaliação conjunta das caívas foram Lauraceae, Myrtaceae, Aquifoliaceae e Sapindaceae, respectivamente, com dez, oito, quatro e três espécies cada uma. As famílias Anacardiaceae, Asteraceae, Euphorbiaceae, Fabaceae, Rosaceae e Rutaceae apresentaram duas espécies cada e as 14 famílias restantes foram representadas por uma única espécie. As famílias com maior densidade na somatória total das cinco áreas foram Myrtaceae (968), Araucariaceae (427), Aquifoliaceae (357) e Lauraceae (274) que juntas representaram $67 \%$ do total de indivíduos amostrados.

As espécies Araucaria angustifolia (pinheiro), Ilex paraguariensis (erva-mate), Myrcia sp. (guamirim), Ocotea porosa (imbuia), Ocotea puberula (canelaguaicá), Drimys brasiliensis (cataia), Ilex microdonta (congonha), Sapium glandulosum (leiteiro), Ilex brevicuspis (caúna) e Cinnamodendron dinisii (pimenteira) apareceram em todas as caívas e, juntas, somaram 2.006 indivíduos ou $66,5 \%$ do total dos 3.016 indivíduos amostrados.

Oliveira \& Rotta (1982), avaliando um povoamento natural de FOM em Colombo, PR, encontraram 103 espécies e 34 famílias, com destaque em número de indivíduos para as famílias Aquifoliaceae, Myrtaceae e Lauraceae. Lingner et al. (2007), em remanescentes de floresta com predominância de pinheiro, medindo indivíduos com DAP acima de 19,09 cm, identificaram 41 espécies distribuídas em 24 famílias botânicas, sendo que as famílias Myrtaceae, Asteraceae, Sapindaceae e Lauraceae abrangiam $34,15 \%$ do número total de indivíduos amostrados. Em São João do Triunfo, PR, Longhi (1980) constatou oito espécies para a família Lauraceae, número muito próximo no obtido no presente trabalho. 
Apesar de terem sido verificadas nas caívas as famílias botânicas predominantes em áreas de povoamento natural de FOM, observa-se que o número de indivíduos arbóreos variou consideravelmente entre as áreas, com destaque para a caíva C5 com apenas 217 indivíduos.

Apesar da ação antrópica por períodos relativamente longos, ainda há manutenção das principais famílias botânicas características de FOM nas caívas. Essa informação contribui para que essas áreas sejam consideradas como importantes formações florestais secundárias, cujos recursos devem contribuir para o desenvolvimento rural e a conservação ambiental na região do Planalto Norte Catarinense. Seu papel na conservação ambiental da região pode ocorrer, por exemplo, através de seu uso em programas de corredores ecológicos, aliando produção animal e certa manutenção de serviços ambientais.

A Tabela 2 apresenta os resultados de diversidade, equabilidade, número de espécies e área basal das cinco caívas. O índice de diversidade (H'), cujos valores variaram de 2,13 a 3,30 nats ind $^{-1}$ entre as caívas, indica alta diversidade, comparável ao valor obtido por Cordeiro \& Rodrigues (2007) em um remanescente de FOM em Guarapuava, PR. O índice de equabilidade de Pielou permitiu verificar que os indivíduos encontravamse uniformemente distribuídos nas cinco caívas analisadas. Com relação à área basal, ocorrem valores diferenciados entre as áreas, variando de 8,27 a 19,88 $\mathrm{m}^{2}$ ha $^{-1}$. As caívas 1, 3 e 4 apresentaram valores próximos de área basal, podendo, por este critério, serem classificadas como em estágio avançado de regeneração (entre $15 \mathrm{~m}^{2} \mathrm{e}$ $20 \mathrm{~m}^{2}$ de área basal) de acordo com as resoluções Conama 004/1994 e Conama 38/2007 (Conselho..., 2008) que definem estágios sucessionais para a FOM no Estado de Santa Catarina.
Tabela 2. Valores de índice de diversidade de Shannon $\left(\mathrm{H}^{\prime}\right)$, equabilidade de Pielou (J'), número de espécies ha-1 $(\mathrm{S})$ e área basal para cinco áreas de caíva no Planalto Norte de Santa Catarina.

\begin{tabular}{ccccc}
\hline \multirow{2}{*}{ Caíva } & \multicolumn{1}{c}{$\mathbf{H}$} & $\mathbf{J}$ & $\mathbf{S}$ & Área basal \\
\cline { 2 - 5 } & \multicolumn{2}{c}{ nats ind $^{\mathbf{1}}$} & unidade & $\mathbf{m}^{\mathbf{2}} \mathbf{h a}^{\mathbf{- 1}}$ \\
\hline 1 & 3,309 & 0,8852 & 42 & 19,03 \\
2 & 2,133 & 0,738 & 18 & 8,27 \\
3 & 2,829 & 0,8491 & 28 & 15,74 \\
4 & 2,742 & 0,7843 & 33 & 19,88 \\
5 & 2,813 & 0,8536 & 27 & 9,46 \\
\hline
\end{tabular}

A similaridade entre as caívas, calculada pelo índice de Sorensen, apresentou valores elevados entre elas, sendo que apenas a caíva 2 apresentou valores menores em comparação com as demais, no entanto, todos acima de $50 \%$. A menor similaridade ocorreu entre as caívas 1 e 2 e 2 e 5 (Tabela 3 ).

Tabela 3. Matriz de similaridade com o uso do índice de Sorensen para cinco áreas de caíva no Planalto Norte de Santa Catarina.

\begin{tabular}{clllll}
\hline Caíva & $\mathbf{1}$ & $\mathbf{2}$ & $\mathbf{3}$ & $\mathbf{4}$ & $\mathbf{5}$ \\
\hline 1 & 1,00 & 0,53 & 0,71 & 0,72 & 0,72 \\
2 & & 1,00 & 0,61 & 0,63 & 0,53 \\
3 & & & 1,00 & 0,70 & 0,70 \\
4 & & & & 1,00 & 0,63 \\
5 & & & & & 1,00 \\
\hline
\end{tabular}

A relação das espécies com seus respectivos valores médios e desvio padrão dos parâmetros fitossociológicos está apresentada na Tabela 4, ordenada de acordo com o valor de importância (VI). 
Tabela 4. Valores médios ( $\mu$ ) e desvio padrão (s) dos parâmetros fitossociológicos valor de importância (VI), densidade absoluta (DA), densidade relativa (DR), frequência absoluta (FA), frequência relativa (FR), dominância absoluta (DoA) e dominância relativa (DoR) de espécies botânicas identificadas em cinco caívas no Planalto Norte de Santa Catarina.

\begin{tabular}{|c|c|c|c|c|c|c|c|c|c|c|c|c|c|c|}
\hline \multirow{2}{*}{ Espécies } & \multicolumn{2}{|c|}{ VI } & \multicolumn{2}{|c|}{ DA } & \multicolumn{2}{|c|}{ DR } & \multicolumn{2}{|c|}{ FA } & \multicolumn{2}{|c|}{ FR } & \multicolumn{2}{|c|}{ DoA } & \multicolumn{2}{|c|}{ DoR } \\
\hline & $\mu$ & $\mathbf{s}$ & $\mu$ & $\mathbf{s}$ & $\mu$ & $\mathbf{s}$ & $\mu$ & $\mathbf{s}$ & $\mu$ & $\mathbf{s}$ & $\mu$ & $\mathbf{s}$ & $\mu$ & $\mathbf{s}$ \\
\hline Myrcia sp. & 45,28 & 48,84 & 157,8 & 156,9 & 27,07 & 31,25 & 84,0 & 16,73 & 5,81 & 2,65 & 1,39 & 1,40 & 12,39 & 15,31 \\
\hline Araucaria angustifolia & 44,91 & 33,39 & 85,40 & 93,48 & 14,79 & 15,30 & 92,0 & 17,89 & 6,11 & 2,18 & 3,63 & 4,16 & 24,01 & 19,17 \\
\hline Ocotea porosa & 22,47 & 21,51 & 29,40 & 29,53 & 7,37 & 11,08 & 65,0 & 27,84 & 4,21 & 2,35 & 1,39 & 0,72 & 10,89 & 8,22 \\
\hline $\begin{array}{l}\text { Cinnamodendron } \\
\text { dinisii }\end{array}$ & 20,34 & 6,23 & 41,80 & 24,27 & 7,14 & 2,31 & 96,0 & 8,94 & 6,54 & 2,43 & 0,89 & 0,55 & 6,65 & 3,58 \\
\hline Ilex paraguariensis & 13,69 & 7,89 & 43,20 & 51,10 & 6,93 & 6,06 & 59,0 & 23,56 & 4,37 & 2,65 & 0,33 & 0,31 & 2,40 & 1,97 \\
\hline Cupania vernalis & 12,48 & 12,87 & 15,20 & 15,45 & 2,03 & 1,83 & 64,0 & 49,80 & 3,49 & 2,61 & 1,17 & 1,43 & 6,96 & 8,96 \\
\hline $\begin{array}{l}\text { Sebastiania } \\
\text { commersoniana }\end{array}$ & 8,73 & 7,95 & 18,00 & 23,58 & 2,29 & 2,25 & 48,0 & 41,47 & 2,72 & 2,26 & 0,60 & 0,69 & 3,72 & 3,96 \\
\hline Ilex brevicuspis & 8,52 & 3,59 & 14,40 & 19,81 & 1,92 & 1,82 & 74,0 & 19,49 & 4,76 & 1,19 & 0,30 & 0,35 & 1,84 & 1,79 \\
\hline Drimys brasiliensis & 8,00 & 3,98 & 14,80 & 17,30 & 2,24 & 1,86 & 62,0 & 17,89 & 4,20 & 1,83 & 0,20 & 0,14 & 1,56 & 1,38 \\
\hline Clethra scabra & 7,95 & 10,05 & 17,40 & 32,28 & 2,01 & 3,17 & 44,0 & 35,78 & 2,41 & 1,52 & 0,65 & 1,12 & 3,53 & 5,85 \\
\hline Ilex microdonta & 7,91 & 3,40 & 8,40 & 8,99 & 1,49 & 1,29 & 69,0 & 28,37 & 4,18 & 1,03 & 0,34 & 0,36 & 2,25 & 1,82 \\
\hline Casearia decandra & 7,36 & 7,36 & 17,60 & 20,38 & 2,56 & 2,85 & 52,0 & 41,47 & 2,93 & 2,36 & 0,31 & 0,40 & 1,86 & 2,51 \\
\hline Schinus terebinthifolius & 6,63 & 7,87 & 21,40 & 42,32 & 2,46 & 4,23 & 42,0 & 37,68 & 2,59 & 1,93 & 0,26 & 0,46 & 1,58 & 2,41 \\
\hline $\begin{array}{l}\text { Campomanesia } \\
\text { xanthocarpa }\end{array}$ & 6,50 & 5,49 & 12,80 & 14,29 & 2,05 & 2,67 & 60,0 & 46,90 & 3,26 & 2,38 & 0,19 & 0,19 & 1,20 & 1,19 \\
\hline $\begin{array}{l}\text { Lonchocarpus } \\
\text { subglaucescens }\end{array}$ & 5,64 & 7,17 & 6,60 & 8,17 & 1,81 & 2,97 & 36,0 & 35,78 & 2,26 & 2,46 & 0,20 & 0,27 & 1,57 & 2,10 \\
\hline Mosiera prismática & 5,20 & 11,03 & 16,00 & 34,67 & 2,99 & 6,57 & 24,0 & 43,36 & 1,34 & 2,56 & 0,17 & 0,38 & 0,87 & 1,91 \\
\hline Sapium glandulatum & 5,02 & 4,35 & 4,40 & 4,22 & 0,69 & 0,50 & 43,0 & 24,39 & 2,99 & 2,47 & 0,14 & 0,12 & 1,35 & 1,51 \\
\hline $\begin{array}{l}\text { Cinnamomum } \\
\text { vesiculosum }\end{array}$ & 4,86 & 4,16 & 6,20 & 7,16 & 0,96 & 1,09 & 44,0 & 38,47 & 2,41 & 1,74 & 0,27 & 0,33 & 1,49 & 1,61 \\
\hline $\begin{array}{l}\text { Cryptocarya } \\
\text { aschersoniana }\end{array}$ & 4,47 & 9,99 & 2,20 & 4,92 & 0,48 & 1,07 & 20,0 & 44,72 & 1,95 & 4,36 & 0,17 & 0,38 & 2,04 & 4,56 \\
\hline Cydonia oblonga & 4,43 & 5,18 & 5,40 & 8,41 & 0,70 & 0,87 & 48,0 & 50,20 & 3,23 & 4,03 & 0,07 & 0,11 & 0,50 & 0,65 \\
\hline $\begin{array}{l}\text { Nectandra } \\
\text { megapotamica }\end{array}$ & 3,84 & 5,85 & 9,20 & 18,94 & 1,15 & 1,90 & 28,0 & 43,82 & 1,47 & 2,03 & 0,21 & 0,40 & 1,22 & 2,08 \\
\hline Lithraea brasiliensis & 3,56 & 3,95 & 7,60 & 10,26 & 1,18 & 1,78 & 29,0 & 33,24 & 1,60 & 1,50 & 0,15 & 0,23 & 0,77 & 1,13 \\
\hline Allophylus edulis & 3,25 & 2,61 & 4,00 & 4,64 & 0,48 & 0,48 & 41,0 & 36,81 & 2,31 & 1,82 & 0,06 & 0,06 & 0,47 & 0,43 \\
\hline Ocotea corymbosa & 3,16 & 4,97 & 3,20 & 5,07 & 0,55 & 0,97 & 28,0 & 33,47 & 1,57 & 2,00 & 0,20 & 0,42 & 1,04 & 2,11 \\
\hline Ocotea puberula & 3,08 & 1,12 & 1,60 & 0,89 & 0,29 & 0,12 & 29,0 & 10,25 & 1,85 & 0,53 & 0,11 & 0,06 & 0,94 & 0,79 \\
\hline Ocotea $\mathrm{sp}$ & 3,01 & 5,29 & 2,40 & 3,58 & 0,53 & 0,81 & 16,0 & 26,08 & 1,16 & 2,17 & 0,14 & 0,22 & 1,29 & 2,33 \\
\hline Zanthoxylum sp. & 2,89 & 2,05 & 4,00 & 3,32 & 0,54 & 0,41 & 33,0 & 26,36 & 2,06 & 1,60 & 0,05 & 0,04 & 0,29 & 0,22 \\
\hline Zanthoxylum rhoifolium & 2,84 & 3,89 & 4,60 & 6,54 & 0,63 & 0,86 & 36,0 & 49,80 & 1,75 & 2,40 & 0,09 & 0,14 & 0,46 & 0,75 \\
\hline Ilex theezans & 2,79 & 4,72 & 5,40 & 11,52 & 0,62 & 1,15 & 24,0 & 43,36 & 1,14 & 1,77 & 0,18 & 0,35 & 1,02 & 1,82 \\
\hline
\end{tabular}


Tabela 2. Continuação.

\begin{tabular}{|c|c|c|c|c|c|c|c|c|c|c|c|c|c|c|}
\hline \multirow{2}{*}{ Espécies } & \multicolumn{2}{|c|}{ VI } & \multicolumn{2}{|c|}{ DA } & \multicolumn{2}{|c|}{ DR } & \multicolumn{2}{|c|}{ FA } & \multicolumn{2}{|c|}{ FR } & \multicolumn{2}{|c|}{ DoA } & \multicolumn{2}{|c|}{ DoR } \\
\hline & $\mu$ & $\mathbf{s}$ & $\mu$ & $\mathbf{s}$ & $\mu$ & $\mathbf{s}$ & $\mu$ & $\mathbf{s}$ & $\mu$ & $\mathbf{s}$ & $\mu$ & $\mathbf{s}$ & $\mu$ & $\mathbf{s}$ \\
\hline Jacaranda puberula & 2,72 & 2,37 & 2,40 & 1,67 & 0,47 & 0,38 & 28,0 & 17,89 & 1,70 & 1,25 & 0,07 & 0,08 & 0,56 & 0,86 \\
\hline Rollinia rugulosa & 2,44 & 2,71 & 4,20 & 6,72 & 0,59 & 0,79 & 22,0 & 22,80 & 1,68 & 2,04 & 0,02 & 0,03 & 0,17 & 0,21 \\
\hline Eugenia uniflora & 2,14 & 2,49 & 3,80 & 5,22 & 0,84 & 1,19 & 16,0 & 16,73 & 0,89 & 0,84 & 0,05 & 0,06 & 0,41 & 0,63 \\
\hline Acnistus breviflorus & 2,06 & 1,93 & 1,20 & 1,30 & 0,18 & 0,21 & 33,0 & 41,17 & 1,76 & 1,74 & 0,02 & 0,04 & 0,11 & 0,22 \\
\hline Eugenia uvalha & 1,48 & 2,66 & 1,80 & 2,68 & 0,21 & 0,32 & 20,0 & 34,64 & 1,10 & 2,04 & 0,03 & 0,05 & 0,17 & 0,31 \\
\hline Vitex megapotamica & 1,37 & 1,80 & 1,40 & 1,67 & 0,44 & 0,79 & 12,0 & 10,95 & 0,73 & 0,73 & 0,02 & 0,03 & 0,20 & 0,37 \\
\hline Rapanea umbellata & 1,06 & 1,88 & 1,40 & 2,61 & 0,25 & 0,50 & 12,0 & 17,89 & 0,63 & 1,02 & 0,04 & 0,08 & 0,18 & 0,38 \\
\hline Cedrela fissilis & 1,05 & 2,35 & 0,60 & 1,34 & 0,11 & 0,25 & 4,0 & 8,94 & 0,24 & 0,53 & 0,14 & 0,31 & 0,70 & 1,57 \\
\hline Guapira opposita & 0,68 & 1,03 & 0,40 & 0,55 & 0,11 & 0,20 & 8,0 & 10,95 & 0,49 & 0,74 & 0,01 & 0,02 & 0,07 & 0,10 \\
\hline Prunus sellowii & 0,67 & 0,94 & 0,80 & 1,30 & 0,10 & 0,14 & 8,0 & 10,95 & 0,40 & 0,56 & 0,03 & 0,07 & 0,18 & 0,38 \\
\hline Matayba elaeagnoides & 0,67 & 1,50 & 0,20 & 0,45 & 0,09 & 0,21 & 4,0 & 8,94 & 0,33 & 0,75 & 0,02 & 0,05 & 0,25 & 0,55 \\
\hline Syagrus romanzoffiana & 0,65 & 1,44 & 0,40 & 0,89 & 0,08 & 0,17 & 8,0 & 17,89 & 0,47 & 1,05 & 0,02 & 0,04 & 0,10 & 0,22 \\
\hline $\begin{array}{l}\text { Tabebuia avellanedae } \\
\text { var. paulensis }\end{array}$ & 0,57 & 1,28 & 0,40 & 0,89 & 0,08 & 0,17 & 8,0 & 17,89 & 0,47 & 1,05 & 0,00 & 0,01 & 0,02 & 0,06 \\
\hline Myrcia laruotteana & 0,50 & 0,72 & 0,80 & 1,30 & 0,09 & 0,16 & 8,0 & 10,95 & 0,40 & 0,56 & 0,00 & 0,00 & 0,01 & 0,02 \\
\hline Persea venosa & 0,50 & 1,11 & 0,20 & 0,45 & 0,09 & 0,21 & 4,0 & 8,94 & 0,33 & 0,75 & 0,01 & 0,02 & 0,07 & 0,16 \\
\hline Psidium coriaceum & 0,43 & 0,96 & 0,40 & 0,89 & 0,08 & 0,17 & 4,0 & 8,94 & 0,24 & 0,53 & 0,02 & 0,05 & 0,12 & 0,27 \\
\hline Acacia plumosa & 0,26 & 0,59 & 0,20 & 0,45 & 0,02 & 0,05 & 4,0 & 8,94 & 0,24 & 0,53 & 0,00 & 0,00 & 0,00 & 0,01 \\
\hline Vernonia discolor & 0,26 & 0,59 & 0,20 & 0,45 & 0,02 & 0,05 & 4,0 & 8,94 & 0,24 & 0,53 & 0,00 & 0,00 & 0,00 & 0,01 \\
\hline Terminalia australis & 0,23 & 0,52 & 0,60 & 1,34 & 0,06 & 0,14 & 4,0 & 8,94 & 0,16 & 0,36 & 0,00 & 0,00 & 0,01 & 0,03 \\
\hline Eugenia involucrata & 0,22 & 0,48 & 0,20 & 0,45 & 0,02 & 0,05 & 4,0 & 8,94 & 0,16 & 0,36 & 0,01 & 0,01 & 0,03 & 0,08 \\
\hline Nectandra grandiflora & 0,21 & 0,48 & 0,20 & 0,45 & 0,02 & 0,05 & 4,0 & 8,94 & 0,16 & 0,36 & 0,01 & 0,01 & 0,03 & 0,07 \\
\hline $\begin{array}{l}\text { Cinnamomum } \\
\text { sellowianum }\end{array}$ & 0,19 & 0,42 & 0,20 & 0,45 & 0,02 & 0,05 & 4,0 & 8,94 & 0,16 & 0,36 & 0,00 & 0,00 & 0,01 & 0,01 \\
\hline
\end{tabular}

Considerando a média das cinco caívas, apenas três espécies - o guamirim (Myrcia sp.), o pinheiro (Araucaria angustifolia) e a erva-mate (Ilex paraguariensis) apresentaram valores médios de densidade absoluta acima de 50 indivíduos por hectare, embora a imbuia (Ocotea porosa) também tenha apresentado essa característica em três caívas. A erva-mate (Ilex paraguariensis) contribuiu com os maiores valores de densidade em três das cinco caívas, apesar da grande variação do número de indivíduos por hectare entre elas. Oliveira \& Rotta (1982), analisando a estrutura horizontal de uma Mata de Araucária em Colombo, PR, verificaram que a erva-mate foi a espécie que apresentou a maior densidade, com 62 árvores por hectare.
Com relação à frequência, 11 espécies se destacaram por apresentarem distribuição acima de $50 \%$ entre as caívas, entre elas Ilex paraguariensis, que sempre teve grande importância econômica para a região devido à comercialização de suas folhas para a fabricação de chimarrão e chá-mate. Com relação ao valor de importância (VI), o pinheiro (Araucaria angustifolia) e o guamirim (Myrcia sp.) destacaram-se dos demais pelos altos valores encontrados, o que se deve, em parte, aos altos valores de densidade e frequência dessas espécies. As espécies com maiores VI encontradas neste trabalho se repetem entre as mais abundantes em outros levantamentos realizados em áreas de FOM (Oliveira \& Rotta, 1982; Negrelle \& Silva, 1992; Seger et al., 
2005; Cordeiro \& Rodrigues, 2007; Lingner et al., 2007), contribuindo para a caracterização das caívas como remanescentes florestais, apesar da interferência antrópica e da presença constante de animais por períodos relativamente longos.

É importante destacar que os dados deste estudo envolveram espécies lenhosas com DAP igual ou superior a $5 \mathrm{~cm}$, não sendo considerados indivíduos de menor diâmetro ou espécies herbáceas que poderiam indicar um grau mais preciso da capacidade de regeneração das caívas frente ao pastejo. Visualmente, pode-se constatar que a regeneração das caívas é menor do que em áreas de mata nativa sem pastoreio, sendo que este trabalho está sendo desenvolvido em parcelas específicas para o estudo de regeneração.

\section{Conclusão}

As caívas estudadas apresentam elevada riqueza florística e considerável grau de conservação como remanescentes de Floresta Ombrófila Mista, apesar da interferência antrópica e da presença constante de animais. No entanto, a variação do estado de conservação entre as caívas remete para a necessidade de estudos do efeito das ações antrópicas, em especial em relação à regeneração das espécies existentes.

\section{Referências}

ANGIOSPERM PHYLOGENY GROUP II. An update of the Angiosperm Phylogeny Group classification for orders and families of flowering plants: APG II. Botanical Journal of the Linnean Society, v. 141, p. 399-436, 2003.

BACKES, P.; IRGANG, B. Árvores do Sul: Guia de identificação e interesse ecológico. Instituto Souza Cruz, 2004. 326 p.

CHANG, M. Y. Sistema faxinal: uma forma de organização camponesa em desagregação no centro-sul do Paraná.

Londrina: IAPAR, 1988. 123 p. (IAPAR. Boletim Tecnico, 22).

CONSELHO NACIONAL DO MEIO AMBIENTE. Resoluções

do Conama: resoluções vigentes publicadas entre julho de 1984 e novembro de 2008 - 2. ed. / Conselho Nacional do Meio Ambiente. - Brasília: Conama, 2008. 928 p.

CORDEIRO, J.; RODRIGUES, W. A. Caracterização fitossociológica de um remanescente de Floresta Ombrófila Mista em Guarapuava/PR. Revista Árvore [online]. v. 31, n. 3, p. 545554. 2007. doi: 10.1590/S0100-67622007000300020.

HANISCH, A. L.; MARQUES, A. C.; BONA, L. C. Resposta de pastagens nativas à adubação com insumos agroecológicos em áreas de caíva no Planalto Norte Catarinense. R.E.V.I. Revista de Estudos do Vale Iguaçu, v. 14, p. 139-148, 2009
LAMPRECHT, H. Ensayo sobre la estrutura floristica de la parte sur - oriental del bosque universitario "El Caimital", Estado Barinas. Revista Forestal Venezolana, Mérida, v. 7, n. 10/11, p. 77-119, 1964.

LINGNER, D. V.; OLIVEIRA, Y. M. M. de; ROSOT, N. C.; DLUGOSZ, F. L. Caracterização da estrutura e dinâmica de um remanescente de Floresta com Araucária no Planalto Catarinense. Pesquisa Florestal Brasileira, n. 55, p. 55-66, 2007.

LONGHI, S. J. A estrutura de uma floresta natural de Araucaria angustifolia (Bert.) O. Ktze, no sul do Brasil. 1980. 198 f. Dissertação (Mestrado em Ciências) - Curso de Pós-Graduação em Engenharia Florestal, Setor de Ciências Agrárias, Universidade Federal do Paraná, Curitiba.

MAGURRAN, A. E. Ecological diversity and its measurement. Princeton: Princeton University Press, 1988. 179 p.

MISSOURI BOTANICAL GARDEN'S. W3Most Nomenclature database. Disponível em <http://mobot.mobot.org/W3T/Search/ vast.html>. Acesso em: 19 abr. 2010.

MUELLER-DOMBOIS, D.; ELLENBERG, H. Aims and methods of vegetation ecology. New York: J. Wiley, 1974. 547 p

NEGRELLE, R. A. B; SILVA, F. C. da. Fitossociologia de um trecho de floresta com Araucaria angustifolia (Bert) O. Ktze. no município de Caçador-SC. Boletim de Pesquisa Florestal, n. 24/25, p. 37-54, 1992.

OLIVEIRA, Y. M. M. de; ROTTA, E. Levantamento da estrutura horizontal de uma Mata de Araucária do Primeiro Planalto Paranaense. Boletim de Pesquisa Florestal, n. 4, p. 1-46, jun. 1982.

PIMENTEL, D.; STACHOW, U.; TAKACS, D. A.; BRUBAKER, H. W.; DUMAS, A. R.; MEANEY, J. J.; O’Neil, J. A. S. ; Onsi, D. E.; CORZILIUS, D. B. Conserving biological diversity in agricultural/ forestry systems. BioScience, v. 42, n. 5, p. 354-362. 1992.

REITZ, R.; KLEIN, R. M.; REIS, A. Projeto Madeira de Santa Catarina. Sudesul/IBDF/Herbário Barbosa Rodrigues. Itajaí, 1978. $320 \mathrm{p}$.

SANQUETTA, C. R.; PIZATTO, W.; PÉLLICO NETTO, S.; FIGUEIREDO FILHO, A.; EISFELD, R. de L. Estrutura vertical de um fragmento de floresta ombrófila mista no Centro-sul do Paraná. Floresta, Curitiba, v. 32, n. 2, p. 267-276, jul./dez. 2002.

SEGER, C. D.; DLUGOSZ, F. L.; KURASZ, G. Levantamento florístico e análise fitossociológica de um remanescente de Floresta Ombrófila Mista localizada no município de Pinhais, Paraná/Brasil. Floresta, v. 35, n. 2, p. 291-302, 2005

SHEPHERD, G. J. FITOPAC 1.6: Manual do Usuário. Departamento de Botânica, UNICAMP. 2006.

SISTEMA Brasileiro de Classificação de Solos. Brasília, DF: Embrapa Produção de Informação; [Rio de Janeiro]: Embrapa Solos, 1999. Brasília, DF: Embrapa Produção de Informação; [Rio de Janeiro]: Embrapa Solos, 1999. 412 p.

SOUZA, V. C.; LORENZI, H. Botânica Sistemática: guia ilustrado para identificação das famílias de Angiospermas da flora brasileira, baseado em APG II. Nova Odessa: Instituto Plantarum, 2005. 640 p.:il.

Recebido em 27 de abril de 2010 e aprovado em 05 de novembro de 2010 